\title{
Expected Utility and Catastrophic Risk
}

\author{
Masako Ikefuji \\ Roger Laeven² \\ Jan Magnus 3 \\ Chris Muris 4
}

1 University of Southern Denmark, Denmark;

2 University of Amsterdam, the Netherlands;

3 VU University Amsterdam, the Netherlands;

${ }^{4}$ Simon Fraser University, Canada, 
Tinbergen Institute is the graduate school and research institute in economics of Erasmus University Rotterdam, the University of Amsterdam and VU University Amsterdam.

More TI discussion papers can be downloaded at http://www.tinbergen.nl

Tinbergen Institute has two locations:

Tinbergen Institute Amsterdam

Gustav Mahlerplein 117

1082 MS Amsterdam

The Netherlands

Tel.: +31(0)205251600

Tinbergen Institute Rotterdam

Burg. Oudlaan 50

3062 PA Rotterdam

The Netherlands

Tel.: +31(0)10 4088900

Fax: $+31(0) 104089031$

Duisenberg school of finance is a collaboration of the Dutch financial sector and universities, with the ambition to support innovative research and offer top quality academic education in core areas of finance.

DSF research papers can be downloaded at: http://www.dsf.nl/

Duisenberg school of finance

Gustav Mahlerplein 117

1082 MS Amsterdam

The Netherlands

Tel.: +31(0)20 5258579 


\title{
Expected Utility and Catastrophic Risk*
}

\author{
Masako Ikefuji \\ Department of Environmental and Business Economics \\ University of Southern Denmark \\ Roger J. A. Laeven \\ Amsterdam School of Economics \\ University of Amsterdam \\ Jan R. Magnus \\ Department of Econometrics \& Operations Research \\ VU University Amsterdam and Tinbergen Institute \\ Chris Muris \\ Department of Economics \\ Simon Fraser University
}

October 6, 2014

\footnotetext{
${ }^{*}$ We are very grateful to Graciela Chichilnisky, John Einmahl, Reyer Gerlagh, Christian Groth, Johan Eyckmans, Sjak Smulders, Peter Wakker, Aart de Zeeuw, Amos Zemel, and seminar and conference participants at Kyushu University, Tilburg University, the University of Copenhagen, EAERE, and the Tilburg Sustainability Center for helpful comments. This research was funded in part by the Japan Society for the Promotion of Science (JSPS) under grant C-22530177 (Ikefuji) and by the Netherlands Organization for Scientific Research (NWO) under grant Vidi-2009 (Laeven). E-mail addresses: ikefuji@sam.sdu.dk (Ikefuji), R.J.A.Laeven@uva.nl (Laeven), jan@janmagnus.nl (Magnus), cmuris@sfu.ca (Muris).
} 


\title{
Corresponding author:
}

Roger J. A. Laeven

Amsterdam School of Economics

University of Amsterdam

Valckenierstraat 65

1018 XE Amsterdam

The Netherlands

Phone: +31205254219

email: R.J.A.Laeven@uva.nl

\begin{abstract}
An expected utility based cost-benefit analysis is in general fragile to its distributional assumptions. We derive necessary and sufficient conditions on the utility function of the expected utility model to avoid this. The conditions ensure that expected (marginal) utility remains finite also under heavy-tailed distributional assumptions. Our results are context-free and are relevant to many fields encountering catastrophic risk analysis, such as, perhaps most noticeably, insurance and risk management.
\end{abstract}

JEL Classification: D61; D81; G10; G20; Q5.

Keywords: Expected utility; Catastrophe; Cost-benefit analysis; Risk management; Power utility; Exponential utility; Heavy tails. 


\section{Introduction}

An economist, when asked to model decision making under risk or uncertainty for normative purposes, would typically work within the expected utility framework with constant relative risk aversion (that is, power utility). A statistician, on the other hand, would model economic catastrophes through probability distributions with heavy tails. Unfortunately, expected power utility is fragile with respect to heavy-tailed distributional assumptions: expected utility may fail to exist or it may imply conclusions that are 'incredible'.

Economists have long been aware of this tension between the expected utility paradigm and distributional assumptions (Menger, 1934), and the discussions in Arrow (1974), Ryan (1974), and Fishburn (1976) deal explicitly with the trade-off between the richness of the class of utility functions and the generality of the permitted distributional assumptions. Compelling examples in Geweke (2001) corroborate the fragility of the existence of expected power utility with respect to minor changes in distributional assumptions.

The combination of heavy-tailed distributions and the power utility family may not only imply infinite expected utility, but also infinite expected marginal utility, and hence, via the intertemporal marginal rate of substitution (the pricing kernel), lead to unacceptable conclusions in cost-benefit analyses. For example, with heavy-tailed log-consumption and power utility, the representative agent should postpone any unit of current consumption to mitigate future catastrophes. The latter aspect was recently emphasized by Weitzman (2009) in the context of catastrophic climate change. Weitzman also argues that attempts to avoid this unacceptable conclusion will necessarily be non-robust. ${ }^{1}$

\footnotetext{
${ }^{1}$ Related questions about the validity of expected utility analysis in a catastrophic climate change context were analyzed by Chichilnisky (2000) and Tol (2003), and, more recently, by e.g., Horowitz and Lange (2009), Karp (2009), Arrow (2009), Nordhaus (2009, 2011), Pindyck (2011), Buchholz and Schymura (2012), and Chanel and Chichilnisky
} 
In this paper we contribute to this literature on the question of how to conduct expected utility analysis in the presence of catastrophic risks by deriving general theoretical compatibility results on the utility function of the expected utility model, leaving probability distributions unrestricted. Our theoretical results are context-free, and are relevant and allow application to many fields encountering catastrophic risk analysis, such as, perhaps most noticeably, risk management and insurance, but also finance and econometrics, hydrology, meteorology and environmental economics.

\subsection{Four Principles}

Our paper is built on four principles, which will recur in our analysis:

(i) Catastrophic risks are important. To study risks that can lead to catastrophe is important in many areas, e.g., financial (insurer, bank, trader) distress, traffic accidents (bridge collapse, airplane crash, flight control system failure), dike bursts, killer asteroids, nuclear power plant disasters, and extreme climate change. Such low-probability high-impact events should not be ignored in cost-benefit analyses for policy making.

(ii) A good model 'in the center' is not necessarily good 'at the edges'. Suppose we have estimated a function $C=a+b Y$, relating consumption $C$ to disposable income $Y$. The dots in Figure 1 represent the data and the line gives the resulting ordinary least squares prediction $\hat{C}=\hat{a}+\hat{b} Y$. For incomes in the center, roughly between 40 and 80, the consumption function can be well approximated by the regression line. How useful is this result for very low (or very high) incomes? Not very useful. For very low incomes, predicted consumption would be negative! This does not mean that a linear consumption function is useless. But it is only useful in the center of the domain. Models are approximations, not truths (cf. Goovaerts, Kaas and Laeven, 2010, p. 301), and approximations may not work well if we move too far away from the point of approximation. Examples are abundant and easy to find. ${ }^{2}$ In quantitative risk management, it has become common

(2013).

${ }^{2}$ Newton's theory works fine for cars and trains, but not for space ships. Pharmaceutical testing is typically performed on adult men, and may (and often does) work differently for women and children (Litt, 1997). 


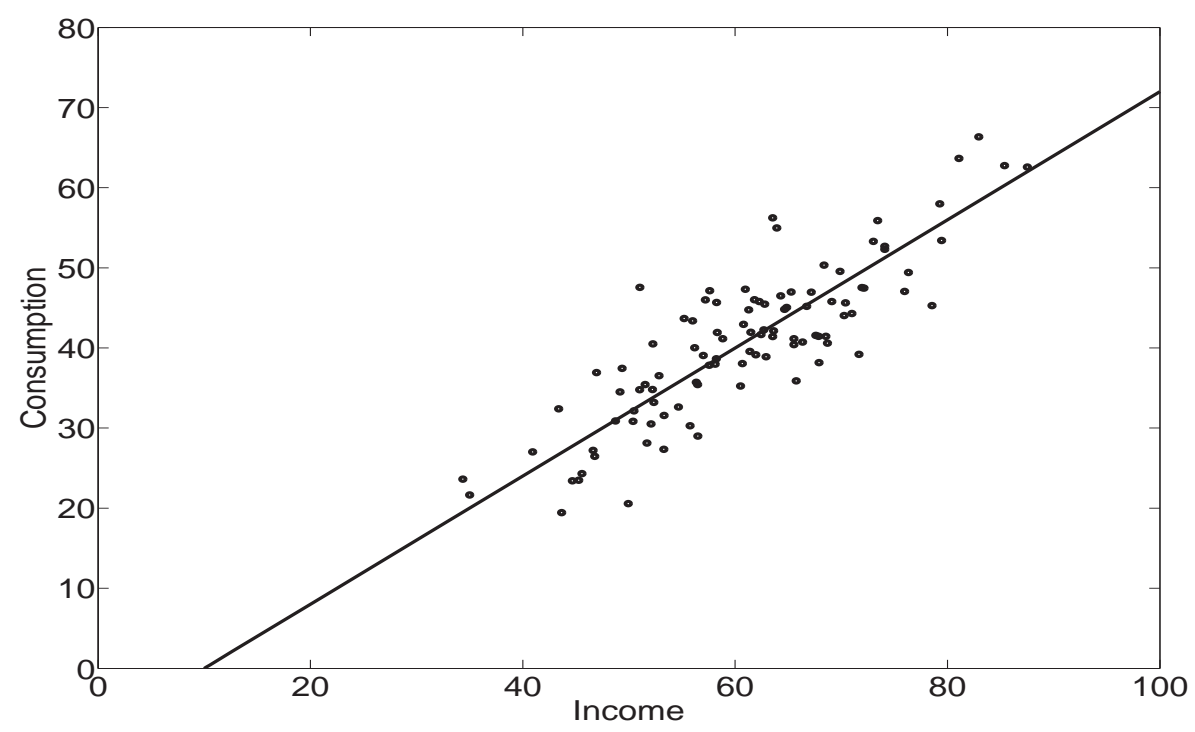

Figure 1: A consumption function

practice to use separate models for the central part of the data and for the extremes, and to 'glue' the models together at a carefully chosen order statistic; see Peng (2001), Johansson (2003) and Necir and Meraghni (2009) and the references therein.

(iii) The price to reduce catastrophic risk is finite. Are we willing to spend all our wealth to avoid children being killed at a dangerous street? Or the dikes to burst? Or a power plant to explode? Or a killer asteroid to hit the Earth? Or climate to change rapidly? No, we are not. To assume the opposite (that a society would be willing to offer all of its current wealth to avoid, mitigate or insurance against catastrophic risks) is not credible, not even from a normative perspective. There is a limit to the amount of current consumption that the representative agent is willing to give up in order to obtain one additional certain unit of future consumption, no matter how extreme and irreversible a catastrophic risk may be. In other words: the expected pricing kernel is finite.

(iv) Light-tailed risks may result in heavy-tailed risk. When $x$ is normally distributed (light tails) then $1 / x$ has no moments (heavy tails). Also, when $x$ is normally distributed then $e^{x}$ has finite moments, but when $x$ follows a Student distribution, then $e^{x}$ has no moments. Even if physical processes do 
not have heavy tails in their distribution, this does not, however, imply that functions of these physical processes cannot have heavy tails. For example, it may well be reasonable to use heavy-tailed distributional assumptions to model future (log) consumption.

\subsection{Contribution and Outline}

Specifically, our analysis delivers necessary and sufficient conditions on the utility function of the expected utility model to avoid fragility of an expected utility based cost-benefit analysis to its distributional assumptions. The conditions we derive ensure that expected utility and expected marginal utility remain finite also under heavy-tailed distributional assumptions, and are context-independent. They guarantee a valid axiomatization of expected utility and avoid incredible consequences in a cost-benefit analysis. We are not aware of other work on this problem in the literature that is of comparable degree of generality.

By leaving distributional assumptions unrestricted, we accommodate principles (i) and (iv) above; by ensuring that expected (marginal) utility remains finite, we account for (iii); and by inspecting the necessary and sufficient conditions we derive, we conclude that the widely adopted power utility function should not be used with catastrophic (heavy-tailed) risks that have non-negligible support 'at the edges', confirming (ii). Instead, the exponential utility (see e.g., Gerber, 1979, Ch. 5, and Goovaerts et al., 2004) or Pareto utility (Ikefuji et al., 2013) are more appropriate.

The remainder of the paper is organized as follows. Section 2 introduces the setting and notation. Section 3 studies expected (marginal) utility and catastrophic risk in a general setting, deriving results on the trade-off between permitted distributional assumptions and the existence of expected (marginal) utility. Section 4 generalizes the main result of Section 3 (to arbitrary order of differentiation). Section 5 concludes. Proofs are relegated to the Appendix. 


\section{Setting and Notation}

We formulate our cost-benefit analysis as a decision under uncertainty problem, in Savage (1954) style. We fix a set $\mathcal{S}$ of states of nature and we let $\mathcal{A}$ denote a $\sigma$-algebra of subsets of $\mathcal{S}$. One state is the true state. We also fix a set $\mathcal{C}$ of consequences (outcomes, consumption) endowed with a $\sigma$-algebra $\mathcal{F}$. Since we are only interested in monetary outcomes, we may take $\mathcal{C}=\mathbb{R}_{+}$. A decision alternative (policy bundle) $X$ is a measurable mapping from $\mathcal{S}$ to $\mathcal{C}$, so that $X^{-1}(A) \in \mathcal{A}$ for all events $A \in \mathcal{F}$. We assume that the class of all decision alternatives $\mathcal{X}$ is endowed with a preference order $\succeq$.

Definition 2.1. We say that expected utility (EU) holds if there exists a measurable and strictly increasing function $U: \mathcal{C} \rightarrow \mathbb{R}$ on the space of consequences, referred to as the utility function, and a probability measure $\mathbb{P}$ on $\mathcal{A}$, such that the preference order $\succeq$ on $\mathcal{X}$ is represented by a functional $V$ of the form $X \mapsto \int_{\mathcal{S}} U(X(s)) d \mathbb{P}=V(X)$. Thus, the decision alternative $X \in \mathcal{X}$ is preferred to the decision alternative $Y \in \mathcal{X}$ if, and only if, $V(X) \geq$ $V(Y)$.

In the Von Neumann and Morgenstern (1944) framework, the utility function $U$ is subjective, whereas the probability measure $\mathbb{P}$ associated with $\mathcal{A}$ is objective and known beforehand (decision under risk). In the more general framework of Savage (1954) adopted here, the probability measure itself can be, but need not be, subjective (decision under uncertainty). We henceforth assume that $U$ is defined for $x \geq 0$, twice differentiable, and such that $U^{\prime}(x)>0$ and $U^{\prime \prime}(x)<0$ for $x>0$.

Since the axiomatization of EU by Von Neumann and Morgenstern (1944) and Savage (1954), numerous objections have been raised against it. These objections relate primarily to empirical evidence that the behavior of agents under risk and uncertainty does not agree with EU. Despite important developments in non-expected utility theory, EU remains the dominant normative decision theory (Broome, 1991; Sims, 2001), and the current paper stays within the framework of EU. Our results presented below provide compatibility conditions under which expected utility theory may reliably provide normatively appealing results, also in the presence of catastrophic 
risks. Of course, one may legitimately question whether EU is the appropriate normative theory for decision making under catastrophic risks and (continue a) search for better theories; see e.g., Chichilnisky (2000). This is, however, beyond our scope.

Definition 2.2. We say that a risk $\epsilon: \mathcal{S} \rightarrow \mathbb{R}$ is heavy-tailed to the left (right) under $\mathbb{P}$ if its moment-generating function is infinite: $\mathrm{E}\left(e^{\gamma \epsilon}\right)=\infty$ for any $\gamma<0(\gamma>0)$.

Examples of heavy-tailed risks are the Student, lognormal, and Pareto distributions. Heavy-tailed risks provide appropriate mathematical models for low-probability high-impact events, such as financial or environmental catastrophes; see e.g., Laeven, Goovaerts and Hoedemakers (2005) and the references therein.

\section{Finite Expected (Marginal) Utility and Heavy Tails}

We state the following result, which dates back to Menger (1934).

Proposition 3.1. If EU is to discriminate univocally among all possible alternative outcome distributions, the utility function must be bounded.

Proposition 3.1 states that the EU functional is finite for all outcome distributions if, and only if, the utility function is bounded. Moreover, it follows as a direct corollary that the axiomatization of $\mathrm{EU}$ is valid for all outcome distributions if, and only if, the utility function is bounded. The implications are non-trivial: boundedness of the utility function must hold not just in exotic situations but also in more familiar and economically relevant settings involving high levels of uncertainty. Only a combination of utility function and outcome distribution that leads to finite expected utility is covered by the axiomatic justification of EU.

Now consider, in a simple one-period setting, a representative agent with time-additive EU preferences and time-preference parameter $\rho>0$. Consumption at time $t=1, C_{1}$, is commonly restricted to a budget-feasible 
consumption set which is subject to uncertainty $\left(\epsilon_{1}\right)$. We assume that the budget restriction takes the generic form

$$
C_{1}^{*}\left(\epsilon_{1}\right) \leq B \exp \left(A \epsilon_{1}\right), \quad B, A>0,
$$

which need not be best-possible and thus accommodates essentially all practical applications. Here $C_{1}^{*}$ is optimal consumption at $t=1$. We exploit (1) to derive compatibility conditions on the utility function.

We normalize without loss of generality the agent's time $t=0$ consumption, $C_{0}$, by setting $C_{0}=1$, and we define the pricing kernel (or intertemporal marginal rate of substitution) as follows:

$$
P\left(C_{1}^{*}\right)=\frac{U^{\prime}\left(C_{1}^{*}\right)}{(1+\rho) U^{\prime}(1)} .
$$

The expectation $\mathrm{E}(P)$ represents the amount of consumption in period 0 that the representative agent is willing to give up in order to obtain one additional certain unit of consumption in period 1 .

Let $\operatorname{RRA}(x)=-x U^{\prime \prime}(x) / U^{\prime}(x)$ and $\operatorname{ARA}(x)=-U^{\prime \prime}(x) / U^{\prime}(x)$ denote relative and absolute risk aversion, respectively, and let

$$
\alpha^{*}=\inf _{x>0} \operatorname{RRA}(x), \quad \beta^{*}=\sup _{x>0} \operatorname{ARA}(x) .
$$

The following result states that the expectation of the pricing kernel is finite for all outcome distributions whenever the concavity index $\operatorname{ARA}(x)$ is bounded.

Proposition 3.2. Assume that EU holds and that the budget feasibility restriction (1) applies.

(a) If $\alpha^{*}>0$ and $\epsilon_{1}$ is heavy-tailed to the left under $\mathbb{P}$, then $\mathrm{E}(P)=\infty$;

(b) If $\beta^{*}<\infty$ and $\alpha^{*}=0$, then $\mathrm{E}(P)<\infty$ for any $\epsilon_{1}$.

If the EU maximizer has decreasing absolute risk aversion and increasing relative risk aversion, as is commonly assumed (Eeckhoudt and Gollier, 1995, Section 4.2, Hypotheses 4.1 and 4.2), a complete and elegant characterization of boundedness of the expected pricing kernel can be obtained, as follows.

Proposition 3.3. Assume that EU holds and that the budget feasibility restriction (1) applies. Assume furthermore that $R R A(x)$ exists and is nonnegative and non-decreasing for all $x \geq 0$ and that $A R A(x)$ is non-increasing 
for all $x>0$. Then, $\mathrm{E}(P)<\infty$ for any $\epsilon_{1}$ if and only if $\int_{0}^{\gamma} A R A(x) d x<\infty$ for some $\gamma>0$.

REMARK 3.1. Notice that, when $\int_{0}^{\gamma} \operatorname{ARA}(x) d x=\infty$ for some $\gamma>0$, both $\alpha^{*}>0$ and $\alpha^{*}=0$ can hold. If $\alpha^{*}>0$ then we do not need the full force of Proposition 3.3; it is sufficient that $\epsilon_{1}$ is heavy-tailed to the left. Then $\mathrm{E}(P)=\infty$ by Proposition 3.2(a). If $\alpha^{*}=0$ then heavy-tailedness alone is not sufficient, but we can always find an $\epsilon_{1}$ such that $\mathrm{E}(P)=\infty$. When $\int_{0}^{\gamma} \operatorname{ARA}(x) d x=\infty$ then $\beta^{*}=\infty$. But when $\int_{0}^{\gamma} \operatorname{ARA}(x) d x<\infty$, both $\beta^{*}<\infty$ and $\beta^{*}=\infty$ can occur.

ExAmple 3.1. An example of an ARA satisfying $\int_{0}^{\gamma} \operatorname{ARA}(x) d x=\infty$ and $\alpha^{*}>0$ is that of power utility. An example of an ARA with $\int_{0}^{\gamma} \operatorname{ARA}(x) d x=$ $\infty$ and $\alpha^{*}=0$ is a function which behaves as $-1 /(x \log x)$ for values of $x$ close to 0 and in addition satisfies the conditions of the proposition.

ExAmple 3.2. An example of an ARA satisfying $\int_{0}^{\gamma} \operatorname{ARA}(x) d x<\infty$ and $\beta^{*}=\infty$ occurs when $\operatorname{ARA}(x)=x^{-\delta}(0<\delta<1)$. An example of an ARA satisfying $\int_{0}^{\gamma} \operatorname{ARA}(x) d x<\infty$ and $\beta^{*}<\infty$ occurs in the case of exponential utility in which $\operatorname{ARA}(x)=\beta(0 \leq \beta<\infty)$, or Pareto utility (Ikefuji et al., 2013). A sufficient condition for $\int_{0}^{\gamma} \operatorname{ARA}(x) d x<\infty$ to hold is that there exists $0 \leq \delta<1$ such that $\lim \sup _{x \downarrow 0} x^{\delta} \operatorname{ARA}(x)<\infty$.

The above propositions provide necessary and sufficient conditions on the utility function to ensure that expected utility and expected marginal utility (hence the expected pricing kernel) are finite, also in the presence of heavy tails. These compatibility results are readily applicable to standard welfare maximization problems. The importance of the results lies in the fact that (a) if (minus) expected utility is infinite, the axiomatic justification of EU is not valid, and (b) if the expected pricing kernel is infinite, then the amount of consumption in period 0 which the representative agent is willing to give up in order to obtain one additional certain unit of consumption in period 1 is infinite, which is not credible in most settings.

REMARK 3.2. Weitzman (2009) recently noted, in a highly stylized setting of extreme climate change, that heavy-tailed uncertainty and power utility are incompatible, since this combination of uncertainty and preferences implies an infinite expected pricing kernel. To avoid this, Weitzman introduces a 
lower bound on consumption, argues that this lower bound is related to a parameter that resembles the value of a statistical life (VSL), and proves that the expected pricing kernel approaches infinity as the value of this parameter approaches infinity (the 'dismal theorem'). Weitzman further argues that this VSL-like parameter is hard to know.

Incompatible pairs of utility functions and distribution functions indeed exist, in the sense that the expected pricing kernel or other important policy variables become infinite. In fact, our results above provide necessary and sufficient conditions on the utility function for the expected pricing kernel to exist, also under heavy tails. But we object to the dismal theorem for the following reason: our results explicate that the dismal theorem is based on an ex ante incompatible (invalid) model specification. It is avoided when the economic model (utility function) is compatible with the statistical model (heavy tails). Note that only then Savage's axiomatization of EU is valid.

\section{Generalization to Arbitrary Order}

Assume $U$ is differentiable for all degrees of differentiation. We denote by $U^{(m)}$ the $m^{\text {th }}$ derivative of $U, m=0,1, \ldots$ Furthermore, assume $(-1)^{m} U^{(m)}(x)<0$, for $x>0$ and $m=0,1, \ldots$ Proposition 3.3 readily generalizes to the following result, valid for any order of differentiation:

Proposition 4.1. Arbitrarily fix $m=0,1, \ldots$ Assume that $E U$ holds and that the budget feasibility restriction (1) applies. Assume furthermore that $-x U^{(m+1)}(x) / U^{(m)}(x)$ exists and is non-negative and non-decreasing for all $x \geq 0$ and that $-U^{(m+1)}(x) / U^{(m)}(x)$ is non-increasing for all $x>0$. Then,

$$
\mathrm{E}\left(U^{(m)}\left(C_{1}^{*}\right) / U^{(m)}(1)\right)<\infty
$$

for any $\epsilon_{1}$ if and only if

$$
\int_{0}^{\gamma}-U^{(m+1)}(x) / U^{(m)}(x) d x<\infty
$$

for some $\gamma>0$.

REMARK 4.1. For $m=0,-U^{(m+1)}(x) / U^{(m)}(x)$ is connected to the (reciprocal of the) fear of ruin index (Foncel and Treich, 2005). For $m=1$, 
$-U^{(m+1)}(x) / U^{(m)}(x)$ is the $\operatorname{ARA}(x)$. For $m=2,-U^{(m+1)}(x) / U^{(m)}(x)$ is the index of absolute prudence (see e.g., Gollier, 2001, p. 265).

REMARK 4.2. For $m=0$, Theorem 4.1 imposes conditions on the (reciprocal of the) fear of ruin index, rather than directly on the utility function, as in Proposition 3.1. In view of the fact that the utility function is a cardinal (or interval) scale, which is unique up to positive affine transformations, one may argue that it is more appealing to consider and restrict the "normalized" function $-U^{(m+1)}(x) / U^{(m)}(x)$.

\section{Concluding Remarks}

We have derived necessary and sufficient conditions in the EU model to avoid fragility of the model to distributional assumptions in an EU based cost-benefit analysis. Based on our generic results regarding the relationship between the richness of the class of utility functions and the generality of the permitted distributional assumptions, applications should restrict to utility functions that are compatible with distributional assumptions. This guarantees a valid axiomatization of EU and avoids the unacceptable conclusion that society should sacrifice an unlimited amount of consumption to reduce the probability of an economic catastrophe by even a small amount.

The analysis in our paper applies to many policy making settings involving catastrophic risks, such as the development of new financial incentive schemes to mitigate the risk of extreme systemic failures and resulting financial economic crises, policies concerning medical risks (pandemic flu and vaccination risks), and economy-climate catastrophe. Furthermore, applications to insurance premium calculation and risk measurement using the equivalent (or zero) utility principle or the certainty equivalent (or mean value) principle (see Goovaerts, De Vylder and Haezendonck, 1984, and Denuit et al., 2006) are straightforward.

In future work, one may analyze the same problem for the dual theory of choice under risk (Yaari, 1987). Also, one could assume more structure on the permitted stochasticity (yet still allow for heavy tails), such as the existence of some moments as in Arrow (1974), in order to broaden the constraints on the utility function. 


\section{Appendix: Proofs}

Proof of Proposition 3.1: See Menger (1934, p. 468) in the context of St.

Petersburg-type lotteries, and also Arrow (1974, p. 136) and Gilboa (2009, pp. 108-109). Menger (implicitly) assumes boundedness from below and demonstrates that boundedness from above should hold, and it is straightforward to generalize his result to an a priori unrestricted setting.

Proof of Proposition 3.2: Let $\alpha^{*}>0$. The EU maximizer is then more risk-averse in the sense of Arrow-Pratt than an agent with power (CRRA) utility of index $\alpha^{*}$. It follows from (2) that

$$
\frac{P^{\prime}\left(C_{1}^{*}\right)}{P\left(C_{1}^{*}\right)}=\frac{U^{\prime \prime}\left(C_{1}^{*}\right)}{U^{\prime}\left(C_{1}^{*}\right)}=-\operatorname{ARA}\left(C_{1}^{*}\right) .
$$

Since $\operatorname{ARA}(x)=\operatorname{RRA}(x) / x \geq \alpha^{*} / x$, we then have

$$
\begin{aligned}
\mathrm{E}(P) & =\frac{1}{1+\rho} \mathrm{E} \exp \left(-\int_{C_{1}^{*}}^{1} d \log P(x)\right)=\frac{1}{1+\rho} \mathrm{E} \exp \left(\int_{C_{1}^{*}}^{1} \operatorname{ARA}(x) d x\right) \\
& \geq \frac{1}{1+\rho} \int_{C_{1}^{*} \leq 1} \exp \left(\int_{C_{1}^{*}}^{1}\left(\alpha^{*} / x\right) d x\right) d F\left(\epsilon_{1}\right) \\
& =\frac{1}{1+\rho} \int_{C_{1}^{*} \leq 1}\left(C_{1}^{*}\right)^{-\alpha^{*}} d F\left(\epsilon_{1}\right) \geq \frac{B^{-\alpha^{*}}}{1+\rho} \int_{C_{1}^{*} \leq 1} e^{-A \alpha^{*} \epsilon_{1}} d F\left(\epsilon_{1}\right)=\infty,
\end{aligned}
$$

using (1) and the fact that $\epsilon_{1}$ is heavy-tailed to the left. This proves part (a). Intuitively, if agent 1 is more risk-averse in the sense of Arrow-Pratt than agent 2 , and if it is optimal to postpone all consumption for agent 2 , then this will also be optimal for agent 1 .

Next let $\alpha^{*}=0$ and $\beta^{*}<\infty$. The EU maximizer is then less risk-averse in the sense of Arrow-Pratt than an agent with exponential (CARA) utility of index $\beta^{*}$. Since $\alpha^{*}=0$, we have $0 \leq \operatorname{ARA}(x) \leq \beta^{*}$ and hence

$$
\begin{aligned}
\mathrm{E}(P)= & \int_{C_{1}^{*} \leq 1} P d F\left(\epsilon_{1}\right)+\int_{C_{1}^{*}>1} P d F\left(\epsilon_{1}\right) \\
\leq & \frac{1}{1+\rho} \int_{C_{1}^{*} \leq 1} \exp \left(\int_{C_{1}^{*}}^{1} \beta^{*} d x\right) d F\left(\epsilon_{1}\right) \\
& +\frac{1}{1+\rho} \int_{C_{1}^{*}>1} \exp \left(-\int_{1}^{C_{1}^{*}} \operatorname{ARA}(x) d x\right) d F\left(\epsilon_{1}\right) \\
\leq & \frac{e^{\beta^{*}} \operatorname{Pr}\left(C_{1}^{*} \leq 1\right)+\operatorname{Pr}\left(C_{1}^{*}>1\right)}{1+\rho}<\infty .
\end{aligned}
$$


Proof of Proposition 3.3: To prove the 'only if' part, we assume that $\int_{0}^{\gamma} \operatorname{ARA}(x) d x$ is infinite for every $\gamma>0$, and then show that there exist $(\mathcal{S}, \mathcal{A}, \mathbb{P})$ and $\epsilon_{1}$ defined on it such that $\mathrm{E}(P)=\infty$. We note that $\beta^{*}=\infty$. Define a function $g:(0,1] \rightarrow[1, \infty)$ by

$$
g(y)=\exp \left(\int_{y}^{1} \operatorname{ARA}(x) d x\right)
$$

Then,

$$
\mathrm{E}(P) \geq \frac{1}{1+\rho} \int_{C_{1}^{*} \leq 1} g\left(\min \left(C_{1}^{*}, 1\right)\right) d F\left(\epsilon_{1}\right) .
$$

Recall from (1) that $C_{1}^{*} \leq B e^{A \epsilon_{1}}$, and let $\epsilon_{1}^{*}$ be such that $B e^{A \epsilon_{1}^{*}}=1$, so that $0<B e^{A \epsilon_{1}} \leq 1$ if and only if $\epsilon_{1} \leq \epsilon_{1}^{*}$. Define $u:(-\infty, \infty) \rightarrow[0, \infty)$ by

$$
u\left(\epsilon_{1}\right)= \begin{cases}g\left(B e^{A \epsilon_{1}}\right)-1 & \text { if } \epsilon_{1} \leq \epsilon_{1}^{*}, \\ 0 & \text { if } \epsilon_{1}>\epsilon_{1}^{*} .\end{cases}
$$

Since $\operatorname{ARA}(1)>0, g$ is monotonically decreasing and we obtain

$$
\begin{array}{r}
\int_{C_{1}^{*} \leq 1} g\left(\min \left(C_{1}^{*}, 1\right)\right) d F\left(\epsilon_{1}\right) \geq \int_{\epsilon_{1} \leq \epsilon_{1}^{*}} g\left(B e^{A \epsilon_{1}}\right) d F\left(\epsilon_{1}\right) \\
=\int_{\epsilon_{1} \leq \epsilon_{1}^{*}}(u+1) d F\left(\epsilon_{1}\right)=\mathrm{E}(u)+\operatorname{Pr}\left(\epsilon_{1} \leq \epsilon_{1}^{*}\right) .
\end{array}
$$

Strict monotonicity of $g$ implies its invertibility. Hence we can choose $u$ to be any non-negative random variable whose expectation does not exist (for example, the absolute value of a Cauchy distribution), and then define $\epsilon_{1}$ through $B_{1} e^{\tau \epsilon_{1}}=g^{-1}(u+1)$. With such a choice of $\epsilon_{1}$ we have $\mathrm{E}(P)=\infty$.

To prove the 'if'-part we assume that $\int_{0}^{\gamma} \operatorname{ARA}(x) d x$ is finite. This implies that $\int_{0}^{1} \operatorname{ARA}(x) d x$ is finite, so that

$$
\begin{aligned}
\mathrm{E}(P)= & \frac{1}{1+\rho} \int_{C_{1}^{*} \leq 1} \exp \left(\int_{C_{1}^{*}}^{1} \operatorname{ARA}(x) d x\right) d F\left(\epsilon_{1}\right) \\
& +\frac{1}{1+\rho} \int_{C_{1}^{*}>1} \exp \left(-\int_{1}^{C_{1}^{*}} \operatorname{ARA}(x) d x\right) d F\left(\epsilon_{1}\right) \\
\leq & \frac{\operatorname{Pr}\left(C_{1}^{*} \leq 1\right)}{1+\rho} \exp \left(\int_{0}^{1} \operatorname{ARA}(x) d x\right)+\frac{\operatorname{Pr}\left(C_{1}^{*}>1\right)}{1+\rho}<\infty,
\end{aligned}
$$

using the fact that $\alpha^{*}=\operatorname{RRA}(0)=0$. 


\section{References}

Arrow, K.J. (1974). The use of unbounded utility functions in expected-utility maximization: Response, Quarterly Journal of Economics, 88, 136-138.

Arrow, K.J. (2009). A note on uncertainty and discounting in models of economic growth, Journal of Risk and Uncertainty, 38, 87-94.

Broome, J.R. (1991). Weighing Goods, Basil Blackwell, Oxford, UK.

Buchholz, W. and M. Schymura (2012). Expected utility theory and the tyranny of catastrophic risks, Ecological Economics, 77, 234-239.

Chanel, O. and G. Chichilnisky (2013). Valuing life: Experimental evidence using sensitivity to rare events, Ecological Economics, 85, 198-205

Chichilnisky, G. (2000). An axiomatic approach to choice under uncertainty with catastrophic risks, Resource and Energy Economics, 22, 221-231.

Denuit, M., J. Dhaene, M.J. Goovaerts, R. Kaas and R.J.A. Laeven (2006). Risk measurement with equivalent utility principles, In: Rüschendorf, L. (Ed.), Risk Measures: General Aspects and Applications, Statistics and Decisions, 24, 1-26.

Eeckhoudt, L., and C. Gollier (1995). Risk: Evaluation, management and sharing. Harvester Wheatsheaf, Hertfordshire.

Fishburn, P.C. (1976). Unbounded utility functions in expected utility theory, Quarterly Journal of Economics, 90, 163-168.

Foncel, J. and N. Treich (2005). Fear of ruin, Journal of Risk and Uncertainty, 31, 289-300.

Gerber, H.U. (1979). An Introduction to Mathematical Risk Theory, S.S. Huebner Foundation Monograph 8, Irwin, Homewood, IL.

Geweke, J. (2001). A note on some limitations of CRRA utility, Economics Letters, 71, $341-345$.

Gilboa, I. (2009). Theory of Decision under Uncertainty, Cambridge University Press, Cambridge, UK.

Gollier, C. (2001). The Economics of Risk and Time, MIT Press, Cambridge, MA.

Goovaerts, M.J., F. De Vylder and J. Haezendonck (1984). Insurance Premiums: Theory and Applications. North-Holland, Amsterdam.

Goovaerts, M.J., R. Kaas, R.J.A. Laeven and Q. Tang (2004). A comonotonic image of independence for additive risk measures, Insurance: Mathematics and Economics, $35,581-594$.

Goovaerts, M.J., R. Kaas and R.J.A. Laeven (2010). Decision principles derived from risk measures, Insurance: Mathematics and Economics, 47, 294-302.

Horowitz, J. and A. Lange (2009). What's wrong with infinity - A note on Weitzman's dismal theorem, Mimeo, University of Maryland. 
Ikefuji, M., R.J.A. Laeven, J.R. Magnus, and C. Muris (2013). Pareto utility, Theory and Decision, 75, 43-57.

Johansson, J. (2003). Estimating the mean of heavy-tailed distributions, Extremes, 6, 91-109.

Karp, L. (2009). Sacrifice, discounting and climate policy: five questions, Mimeo, University of California, Berkeley.

Laeven, R.J.A., M.J. Goovaerts and T. Hoedemakers (2005). Some asymptotic results for sums of dependent random variables, with actuarial applications, Insurance: Mathematics and Economics, 37, 154-172.

Litt, I. (1997). Taking Our Pulse: The Health of American Women, Stanford: Stanford University Press.

Menger, K. (1934). Das Unsicherheitsmoment in der Wertlehre, Zeitschrift für Nationalökonomie, 5, 459-485.

Necir, A. and D. Meraghni (2009). Empirical estimation of the proportional hazard premium for heavy-tailed claim amounts, Insurance: Mathematics and Economics, $45,49-58$.

Nordhaus, W.D. (2009). An analysis of the dismal theorem, Cowles Foundation Discussion Paper 1686, Yale University.

Nordhaus, W.D. (2011). The economics of tail events with an application to climate change, Review of Environmental Economics and Policy, 5, 240-257.

Peng, L. (2001). Estimating the mean of a heavy-tailed distribution, Statistics and Probability Letters, 52, 255-264.

Pindyck, R.S. (2011). Fat tails, thin tails, and climate change policy, Review of Environmental Economics and Policy, 5, 258-274.

Ryan, T.M. (1974). The use of unbounded utility functions in expected-utility maximization: Comment, Quarterly Journal of Economics, 88, 133-135.

Savage, L.J. (1954). The Foundations of Statistics, Wiley, New York, NY.

Sims, C.A. (2001). Pitfalls of a minimax approach to model uncertainty, American Economic Review, 91, 51-54.

Tol, R.S.J. (2003). Is the uncertainty about climate change too large for expected costbenefit analysis?, Climatic Change, 56, 265-289.

Von Neumann, J. and O. Morgenstern $(1944,1947,1953)$. Theory of Games and Economic Behavior, Princeton University Press, Princeton, NJ.

Weitzman, M.L. (2009). On modeling and interpreting the economics of catastrophic climate change, Review of Economics and Statistics, 91, 1-19.

Yaari, M.E. (1987). The dual theory of choice under risk, Econometrica, 55, 95-115. 